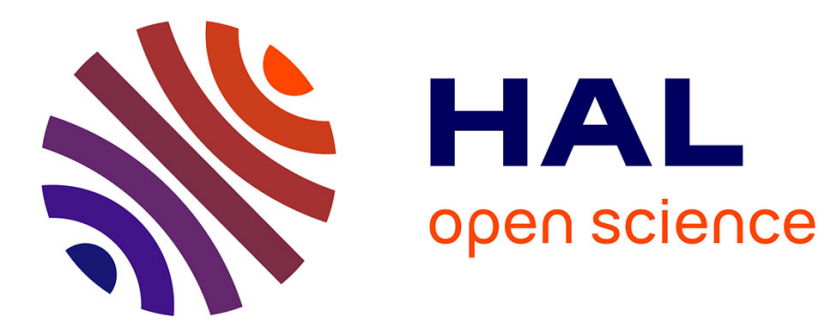

\title{
Automatic formal derivation of the oscillation condition
}

Nicolas Ratier, Laurent Couteleau, Rémi Brendel, Philippe Guillemot

\section{To cite this version:}

Nicolas Ratier, Laurent Couteleau, Rémi Brendel, Philippe Guillemot. Automatic formal derivation of the oscillation condition. International Frequency Control Symposium (IEEE), May 1997, Orlando, Florida, United States. pp.925-931. hal-00345162

\section{HAL Id: hal-00345162 https://hal.science/hal-00345162}

Submitted on 8 Dec 2008

HAL is a multi-disciplinary open access archive for the deposit and dissemination of scientific research documents, whether they are published or not. The documents may come from teaching and research institutions in France or abroad, or from public or private research centers.
L'archive ouverte pluridisciplinaire HAL, est destinée au dépôt et à la diffusion de documents scientifiques de niveau recherche, publiés ou non, émanant des établissements d'enseignement et de recherche français ou étrangers, des laboratoires publics ou privés. 


\title{
AUTOMATIC FORMAL DERIVATION OF THE OSCILLATION CONDITION
}

\author{
N. Ratier, L. Couteleau, R. Brendel, P. Guillemot $\dagger$ \\ Laboratoire de Physique et Métrologie des Oscillateurs du C.N.R.S. \\ associé à l'Université de Franche-Comté-Besançon \\ 32 avenue de l'Observatoire - 25044 Besançon Cedex - France \\ $\dagger$ Centre National d'Etudes Spatiales \\ 18 avenue E. Belin - 31055 Toulouse Cedex - France
}

\begin{abstract}
The behavior of a quartz crystal oscillator can be described by a nonlinear characteristic polynomial whose coefficients are function of the circuit parameters. Solving the polynomial in the frequency domain leads to the steady state oscillation amplitude and frequency. In the time domain, it gives the oscillator signal transient.

Deriving the characteristic polynomial from the circuit description involves lengthy and tedious algebraic calculations if they are performed by hand. They may be now performed by using the symbolic manipulation capabilities of commercially available softwares. However, symbolic analysis using brute force method inevitably leads to an explosion of terms in equations. The paper will present a fully automatic method for generating the coding of an oscillator characteristic polynomial directly from the SPICE description netlist.

The code thus generated is eventually compiled and takes place in an oscillator library. Then it is linked with the numerical main program that solves the polynomials. Solutions to overcome problems related to automatic symbolic calculations are presented and discussed. It is shown that the method used leads to concise and efficient code.
\end{abstract}

\section{Introduction}

Applications such as orbitography or atomic clock processing need more and more stable quartz crystal oscillators. This paper presents the actual state of a computer program especially designed to accurately simulate the behavior of these oscillators. Our program is composed of three distinct parts. Only the first part is exposed in this paper.

- This first part consists in putting into equations the behavior of the oscillator. More precisely, it shows how to build the Fortran program describing the characteristic polynomial starting from the SPICE netlist of the circuit. We have developed a Maple program to complete the topological analysis of the circuit, to process the equations and to generate the Fortran code.

- The second part concerns the numerical calculus of the steady state feature of the oscillator, i.e. its amplitude and frequency. How to represent the nonlinear characteristic of the transistor? How to process the isochronism defect of the resonator? Which algorithms are to be used to compute the roots? These questions are treated in Ref. [1][2].

- The third part concerns the time domain analysis of the oscillator. The characteristic polynomial represents a nonlinear differential equation which modelizes the behavior of the oscillator in the time domain. Because of the high quality factor, this equation is quite difficult to solve. Which method is to be used to solve this equation? Which form the characteristic polynomial has to be given to treat the problem? These questions are analyzed in Ref. [3].

\section{Oscillation condition: Theory}

The transistor is represented [4] by the equivalent circuit as in figure (1). The 4 admittances $Y_{i}, Y_{r}, Y_{f}, Y_{o}$ permit to modelize the nonlinear behavior of the component. Their value depend on the input voltage $u$, on the output voltage $v$ and on the pulsation $\omega(s=j \omega)$ (Eq. 1) . The functions $G_{i}, C_{i}, G_{r}, C_{r}, G_{f}, C_{f}, G_{o}, C_{o}$ are calculated [1] for given bias and temperature conditions by using the electrical simulator SPICE [5][8], they are represented under the form of a table.

$$
\begin{aligned}
Y_{i}(|u|) & =G_{i}(|u|)+s C_{i}(|u|) \\
Y_{r}(|v|) & =G_{r}(|v|)+s C_{r}(|v|) \\
Y_{f}(|u|) & =G_{f}(|u|)+s C_{f}(|u|) \\
Y_{o}(|v|) & =G_{o}(|v|)+s C_{o}(|v|)
\end{aligned}
$$




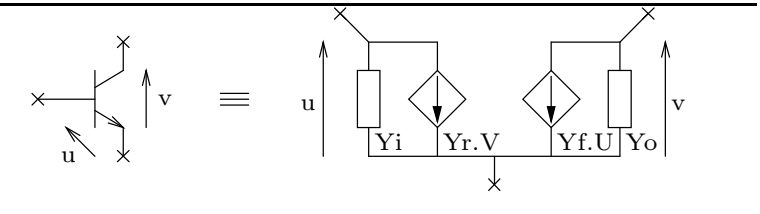

Figure 1: y-parameter representation of the transistor

The main goal is to reduce the circuit of the quartz oscillator to a canonical sketch easily analysable. We have defined 6 transformations (Fig. 2) which allow to reduce progressively the electronic circuit. At each step of the reduction process, a new admittance is integrated into the sketch of the amplifier. The initial amplifier is the transistor itself (Fig. 1).

The y-parameter of the new equivalent circuit are expressed in terms of the initial amplifier component and of the integrated admittance (Table $12^{\text {nd }}$ column). The input and output voltages of the new circuit $\left(U^{\prime}, V^{\prime}\right)$ are expressed in terms of the former ones $(U, V)$. The $4^{\text {th }}$ column of table 1 gives the matrix $A$ which relates the voltages $\left(U^{\prime}, V^{\prime}\right)$ to $(U, V)$, i.e. $\left(U^{\prime}, V^{\prime}\right)^{t}=A(U, V)^{t}$. The initial voltages $(U, V)$ are the voltages $(u, v)$ accross the transistor itself (Fig. 1).

The reduction process of an admittance into the amplifier circuit leads to one of the three configurations relating the quartz to the amplifier. The sketch of these three configurations is given in figure 3. By performing the matrix product of the successive transformations, the voltages $(U, V)$ of the amplifier (Fig 1) are expressed in terms of the transistor input and output voltages $(u, v)$.

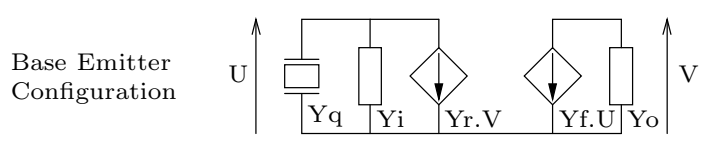

Base Collector Configuration

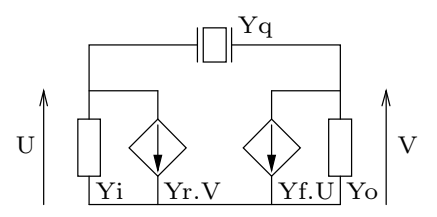

Collector Emitter Configuration

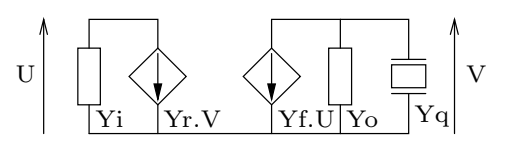

Figure 3: Reduced forms of an oscillator

The application of Kirchhoff's law to both input and output parts of each circuit leads to the three following relations (Eq. 2).

$$
\text { B.E. } \quad \begin{cases}\left(Y_{i}+Y_{q}\right) U+Y_{r} V=0 \\ Y_{f} U & +Y_{o} V=0\end{cases}
$$

$$
\begin{aligned}
& \text { B.C. }\left\{\begin{array}{l}
\left(Y_{i}+Y_{q}\right) U+\left(Y_{r}-Y_{q}\right) V=0 \\
\left(Y_{f}-Y_{q}\right) U+\left(Y_{o}+Y_{q}\right) V=0
\end{array}\right. \\
& \text { C.E. }\left\{\begin{array}{l}
Y_{i} U+Y_{r} V=0 \\
Y_{f} U+\left(Y_{o}+Y_{q}\right) V=0
\end{array}\right.
\end{aligned}
$$

It is important to recall that the three systems of equations (2) are not linear because the transistor yparameters depend on the input or output voltage $u$ or $v$. By equating the ratios $V / U$ in each system of equation, one obtains the relation that should be satisfied by the admittances $Y_{\alpha}$ to ensure the coherence of the systems of Eq. (2).

Table 2: Characteristic polynomial equations

\begin{tabular}{|l|l|}
\hline Configuration & $Y_{i}, Y_{r}, Y_{f}, Y_{o}$ \\
\hline Base Emitter & $\left(Y_{i} Y_{o}-Y_{r} Y_{f}\right)+Y_{o} Y_{q}=0$ \\
\hline Collector Emitter & $\left(Y_{i} Y_{o}-Y_{r} Y_{f}\right)+Y_{i} Y_{q}=0$ \\
\hline Base Collector & $\left(Y_{i} Y_{o}-Y_{r} Y_{f}\right)$ \\
& $+\left(Y_{i}+Y_{r}+Y_{f}+Y_{o}\right) Y_{q}=0$ \\
\hline
\end{tabular}

By replacing each admittance by its numerator and denominator, the equations given in table 1 , that we call "characteristic fraction", take the form (3).

$$
\frac{\sum \prod P}{\sum P}=0
$$

The characteristic polynomial is then $\sum \prod P$. Each term $P$ is expressed under the form of a function of previous relations until reaching the first coefficients which are expressed in terms of the circuit elements $R_{\alpha}, L_{\alpha}, C_{\alpha}$. Equations (2) permit to compute the gain $v / u$ of the oscillator, that is to compute $v$ for a given $u$. When each of the circuit elements is replaced by its numerical value, then the characteristic polynomial is a function of the variables $(u, s=j \omega)$. As an example, the characteristic polynomial of the Colpitts oscillator is plotted in Fig. (4). Each curve represents the imaginary part vs. the real part of the characteristic polynomial with respect to the frequency for different amplitude values.

The numerical calculation of the variables $u$ and $\omega$ which nullify the imaginary part and the real part of the characteristic polynomial determines the frequency and the amplitude of the oscillation. This algorithm uses data calculated by the SPICE program and needs the determination of the first derivative of the characteristic polynomial. The corresponding algorithm used to solve these equations is described in $[2]$.

Because the characteristic polynomial coefficients are expressed as functions of all the circuit components, it is possible for the program to calculate the influence of a change of any component value on the oscillation amplitude and frequency as well as on the resonator excitation level. In the same way, it is possible to get the sensitivity of these quantities to small component variation. 

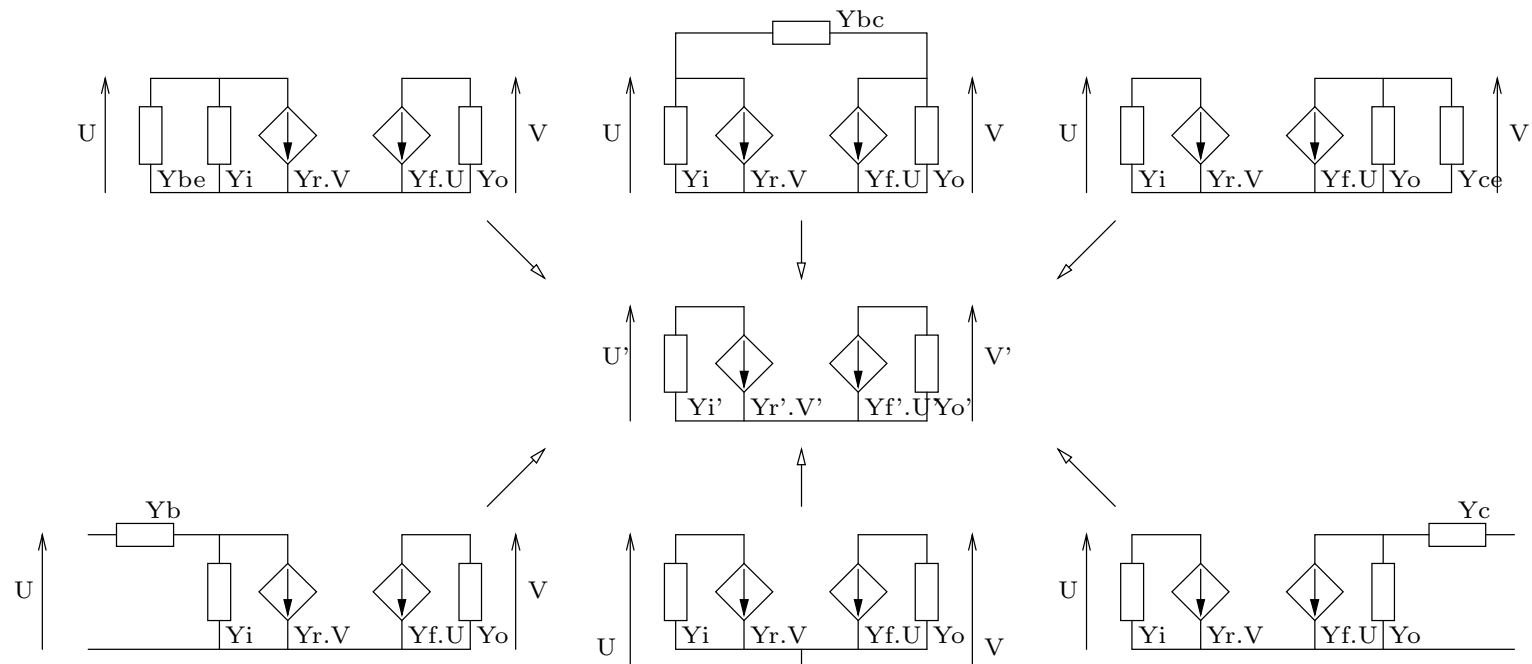

$\mathrm{U}$
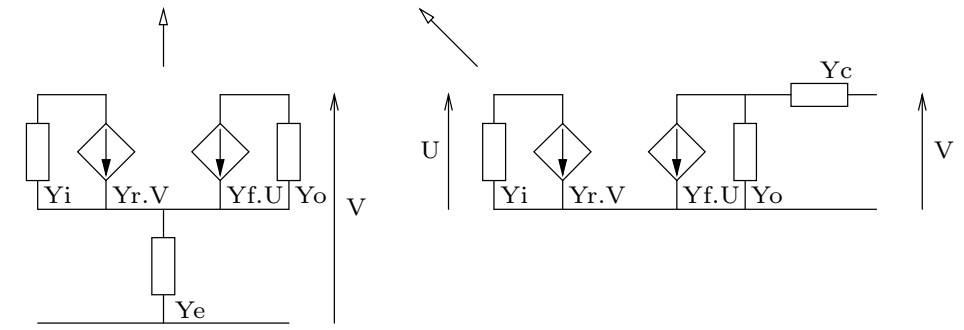

Series Base

Series Emitter

Series Collector

Figure 2: The 6 transformations for circuit reduction

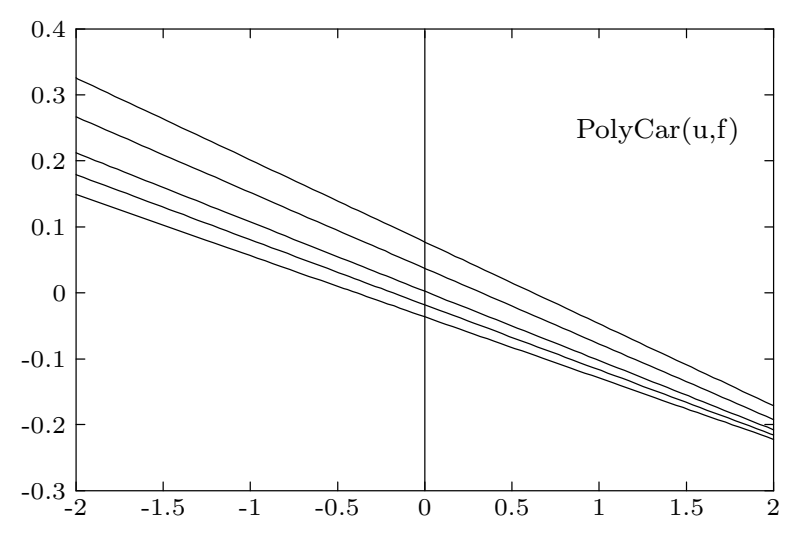

Figure 4: Colpitts oscillator characteristic polynomials

In addition of the rated value the user can specify the tolerance and the temperature coefficient of each component in the input data file. This allows the program to calculate the induced dispersion and to perform worst case analysis. The temperature behavior of the resonator is described by the four coefficients of the frequency temperature curve cubic regression so that the frequency variation can be calculated over a given temperature range.

\section{Oscillation condition: Practice}

Each reduction step defines a set of 4 equations which express the new admittances $Y_{i}, Y_{r}, Y_{f}, Y_{o}$ with respect to the previous ones. In concrete terms, when the oscillator is fully reduced, one obtains a list of equations of the form (4). The functions $f_{i}, f_{r}, f_{f}, f_{o}$ correspond to the equations of the table 1 , the function $g$ corresponds to those of table 2 .

$$
\begin{gathered}
\left\{\begin{array}{c}
Y_{i}^{\prime}=G_{i}+s C_{i} \\
Y_{r}^{\prime}=G_{r}+s C_{r} \\
Y_{f}^{\prime}=G_{f}+s C_{f} \\
Y_{o}^{\prime}=G_{o}+s C_{o} \\
\cdots
\end{array}\right. \\
\left\{\begin{array}{c}
Y_{i}=f_{i}\left(Y_{i}, Y_{r}, Y_{f}, Y_{o}\right) \\
Y_{r}=f_{r}\left(Y_{i}, Y_{r}, Y_{f}, Y_{o}\right) \\
Y_{f}=f_{f}\left(Y_{i}, Y_{r}, Y_{f}, Y_{o}\right) \\
Y_{o}=f_{o}\left(Y_{i}, Y_{r}, Y_{f}, Y_{o}\right)
\end{array}\right.
\end{gathered}
$$

Char Poly $=$ Numerator of $g\left(Y_{i}, Y_{r}, Y_{f}, Y_{o}\right)$

To determine the characteristic polynomial, it is necessary to express the numerator and the denominator of the admittances at each reduction step. How to organize those calculations in practice?

\section{Minimal algebraic expression of the characteristic polynomial}

Writing the equations under the form (4) allows a concise and efficient code [6][7] but may hides possible algebraic simplifications.

- A direct simplification of the polynomial, by deleting all the intermediate variables, leads to the loss of the advantages of the encapsulation method. Moreover, it will take a too long computing time, even in the case of a quite simple oscillator. 
Table 1: Transformation equations

\begin{tabular}{|c|c|c|c|}
\hline & $Y_{i}, Y_{r}, Y_{f}, Y_{o}$ & $Y_{i}, Y_{o}, Y_{s}, \Delta$ & $(U, V)$ \\
\hline $\begin{array}{l}\text { Parallel } \\
\text { Base-Emitter }\end{array}$ & $\begin{aligned} Y_{i}^{\prime} & =Y_{i}+Y_{b e} \\
Y_{r}^{\prime} & =Y_{r} \\
Y_{f}^{\prime} & =Y_{f} \\
Y_{o}^{\prime} & =Y_{o}\end{aligned}$ & $\begin{aligned} Y_{i}^{\prime} & =Y_{i}+Y_{b e} \\
Y_{o}^{\prime} & =Y_{o} \\
Y_{s}^{\prime} & =Y_{s}+Y_{b e} \\
\Delta^{\prime} & =\Delta+Y_{o} Y_{b e}\end{aligned}$ & $\left(\begin{array}{ll}1 & 0 \\
0 & 1\end{array}\right)$ \\
\hline $\begin{array}{l}\text { Parallel } \\
\text { Collector-Emitter }\end{array}$ & $\begin{aligned} Y_{i}^{\prime} & =Y_{i} \\
Y_{r}^{\prime} & =Y_{r} \\
Y_{f}^{\prime} & =Y_{f} \\
Y_{o}^{\prime} & =Y_{o}+Y_{c e}\end{aligned}$ & $\begin{array}{l}Y_{i}^{\prime}=Y_{i} \\
Y_{o}^{\prime}=Y_{o}+Y_{c e} \\
Y_{s}^{\prime}=Y_{s}+Y_{c e} \\
\Delta^{\prime}=\Delta+Y_{i} Y_{c e}\end{array}$ & $\left(\begin{array}{ll}1 & 0 \\
0 & 1\end{array}\right)$ \\
\hline $\begin{array}{l}\text { Parallel } \\
\text { Base-Collector }\end{array}$ & $\begin{array}{l}Y_{i}^{\prime}=Y_{i}+Y_{b c} \\
Y_{r}^{\prime}=Y_{r}-Y_{b c} \\
Y_{f}^{\prime}=Y_{f}-Y_{b c} \\
Y_{o}^{\prime}=Y_{o}+Y_{b c}\end{array}$ & $\begin{array}{l}Y_{i}^{\prime}=Y_{i}+Y_{b c} \\
Y_{o}^{\prime}=Y_{o}+Y_{b c} \\
Y_{s}^{\prime}=Y_{s} \\
\Delta^{\prime}=\Delta+Y_{s} Y_{b c}\end{array}$ & $\left(\begin{array}{ll}1 & 0 \\
0 & 1\end{array}\right)$ \\
\hline $\begin{array}{l}\text { Serie } \\
\text { Base }\end{array}$ & $\begin{array}{l}Y_{i}^{\prime}=\frac{Y_{i} Y_{b}}{Y_{i}+Y_{b}} \\
Y_{r}^{\prime}=\frac{Y_{r} Y_{b}}{Y_{i}+Y_{b}} \\
Y_{f}^{\prime}=\frac{Y_{f} Y_{b}}{Y_{i}+Y_{b}} \\
Y_{o}^{\prime}=\frac{Y_{o} Y_{b}+\left(Y_{i} Y_{o}-Y_{r} Y_{f}\right)}{\left.Y_{j}+Y_{b}\right)}\end{array}$ & $\begin{aligned} Y_{i}^{\prime} & =\frac{Y_{i} Y_{b}}{Y_{i}+Y_{b}} \\
Y_{o}^{\prime} & =\frac{Y_{o} Y_{b}+\Delta}{Y}+Y_{b} \\
Y_{s}^{\prime} & =\frac{Y_{s} Y_{b}+\Delta}{Y_{i}+Y_{b}} \\
\Delta^{\prime} & =\frac{\Delta_{Y} Y_{b}}{Y_{i}+Y_{b}}\end{aligned}$ & $\left(\begin{array}{cc}\frac{Y_{i}+Y_{b}}{Y_{b}} & \frac{Y_{r}}{Y_{b}} \\
0 & 1\end{array}\right.$ \\
\hline $\begin{array}{l}\text { Serie } \\
\text { Collector }\end{array}$ & $\begin{array}{l}Y_{i}^{\prime}=\frac{Y_{i} Y_{c}+\left(Y_{i}+Y_{b}-Y_{r} Y_{f}\right)}{Y_{i}} \\
Y_{r}^{\prime}=\frac{Y_{r} Y_{c}}{Y_{o}+Y_{c}} \\
Y_{f}^{\prime}=\frac{Y_{f} Y_{c}}{Y_{Y}+Y_{c}} \\
Y_{o}^{\prime}=\frac{Y_{o} Y_{c}}{Y_{o}+Y_{c}}\end{array}$ & $\begin{aligned} Y_{i}^{\prime} & =\frac{Y_{i} Y_{c}+\Delta}{Y_{o}+Y_{c}} \\
Y_{o}^{\prime} & =\frac{Y_{o}+Y_{c}}{Y_{O}+Y_{c}} \\
Y_{s}^{\prime} & =\frac{Y_{s} Y_{c}+\Delta}{Y_{o}+Y_{c}} \\
\Delta^{\prime} & =\frac{\Delta Y_{c}}{Y_{o}+Y_{c}}\end{aligned}$ & $\left(\begin{array}{cc}1 & 0 \\
\frac{Y_{f}}{Y_{c}} & \frac{Y_{o}+Y_{c}}{Y_{c}}\end{array}\right)$ \\
\hline $\begin{array}{l}\text { Serie } \\
\text { Emitter }\end{array}$ & $\begin{aligned} Y_{i}^{\prime}=\frac{Y_{o} Y_{c}}{Y_{i}+Y_{e}+\left(Y_{i} Y_{o}-Y_{r} Y_{f}\right)} \\
Y_{r}^{\prime}=\frac{Y_{r} Y_{e}+Y_{f}+Y_{i}+Y_{o}+Y_{e}}{Y_{i}+Y_{r}+Y_{f}+Y_{f}+Y_{r}+Y_{e}} \\
Y_{f}^{\prime}=\frac{Y_{f} Y_{e}-\left(Y_{i} Y_{o}-Y_{r} Y_{f}\right)}{Y_{i}+Y_{r}+Y_{f}+Y_{+}+Y_{e}} \\
Y_{o}^{\prime}=\frac{Y_{o} Y_{e}+\left(Y_{i} Y_{o}-Y_{r} Y_{f}\right)}{Y_{i}+Y_{r}+Y_{f}+Y_{o}+Y_{e}}\end{aligned}$ & $\begin{aligned} Y_{i}^{\prime} & =\frac{Y_{i} Y_{e}+\Delta}{Y_{e}+Y_{e}} \\
Y_{o}^{\prime} & =\frac{Y_{o} Y_{e}+\Delta}{Y_{s}+Y_{e}} \\
Y_{s}^{\prime} & =\frac{Y_{s} Y_{e}}{Y_{s}+Y_{e}} \\
\Delta^{\prime} & =\frac{\Delta Y_{e}}{Y_{s}+Y_{e}}\end{aligned}$ & $\left(\begin{array}{cc}\frac{Y_{i}+Y_{f}+Y_{e}}{Y_{e}} & \frac{Y_{r}+Y_{o}}{Y_{e}} \\
\frac{Y_{i}+Y_{f}}{Y_{e}} & \frac{Y_{r}+Y_{o}+Y_{e}}{Y_{e}}\end{array}\right)$ \\
\hline
\end{tabular}

- The development of a recursive program locating identical expressions would be very difficult, and would have a complexity in time of exponential type.

The transformation equations, table 1 column 2, show the origin of the problem. Each group of transformations have a lot of identical terms and contain "." signs. For example, the expressions of $Y_{i}, Y_{r}, Y_{f}, Y_{o}$ using the series transformation are pretty complex but their sum is rather easy to handle, since a lot of terms vanishes.

\section{Solution}

The problem is solved if there exists a change of variables so that all the transformations only contain "+" signs. This condition guarantees that no subsequent algebraic simplification is possible. This change of variable exists (5) and in addition it gives remarkable properties to the transformation equations. Each variable $Y_{s}$ et $\Delta$ is a function of $|u|$ and $|v|$.

$$
\begin{aligned}
& Y_{i}=Y_{i} \\
& Y_{o}=Y_{o}
\end{aligned}
$$

$$
\begin{aligned}
Y_{s} & =Y_{i}+Y_{r}+Y_{f}+Y_{o} \\
\Delta & =Y_{i} Y_{o}-Y_{r} Y_{f}
\end{aligned}
$$

The transformation equations with the new variables $Y_{i}, Y_{o}, Y_{s}, \Delta$ are given in table 1 , column 3. A careful examination of the equations shows that:

- Those equations only contain "+" signs which is precisely the property looked for.

- The transformation equations using $Y_{i}, Y_{o}, Y_{s}, \Delta$ are more compact than those using $Y_{i}, Y_{r}, Y_{f}, Y_{o}$.

- The transformation equations are divided into two groups: parallel and series. The equations of each group are symmetrical with respect to the admittances $Y_{i}, Y_{o}, Y_{s}$.

With these new variables, the characteristic polynomial expressions are written in Table 3.

One recognize in those last expressions the remarkable properties that we have observed in the transformation equations (Table 1). 
Table 3: Characteristique polynomial equations

\begin{tabular}{|l|l|}
\hline Configuration & $Y_{i}, Y_{o}, Y_{s}, \Delta$ \\
\hline Base Emitter & $\Delta+Y_{o} Y_{q}=0$ \\
\hline Collector Emitter & $\Delta+Y_{i} Y_{q}=0$ \\
\hline Base Collector & $\Delta+Y_{s} Y_{q}=0$ \\
\hline
\end{tabular}

\section{Minimal degree characteristic polyno- mial}

The determination of the oscillator frequency is carried out by computing the root of the characteristic polynomial. The degree of this polynomial is about 20 for an oscillator of medium complexity (12 passive components). The higher the degree the harder the numerical calculus. On the contrary, because the oscillator frequency is close to the resonator frequency, the location of the root is straightforward.

The value of the root is obviously not affected if one multiplies the characteristic polynomial by another arbitrary polynomial . This situation occurs when:

- There is a common factor in the numerator of the characteristic fraction.

- There is a common root in the numerator and denominator of the characteristic fraction.

The direct use of equations (4) leads to a characteristic polynomial with high degree because of these problems. We will show that a suitable representation of the admittance allows to suppress the artificial root of the characteristic polynomial.

\section{Solution}

The transformation equations, table 1 , show that the denominators of each transformation are identical. We will show that this fact implies that, if the variables $Y_{i}, Y_{o}, Y_{s}, \Delta$ are written under the form (6),

$$
Y_{o}=\frac{\frac{N N_{o}}{D N_{o}}}{\frac{N D_{o}}{D D_{o}}}, Y_{i}=\frac{\frac{N N_{i}}{D N_{i}}}{\frac{N D_{i}}{D D_{i}}}, Y_{s}=\frac{\frac{N N_{s}}{D N_{s}}}{\frac{N D_{s}}{D D_{s}}}, \Delta=\frac{\frac{N N_{d}}{D N_{d}}}{\frac{N D_{d}}{D D_{d}}}
$$

then the property (7) occurs.

$$
\begin{aligned}
& N D_{o}=N D_{i}=N D_{s}=N D_{d} \\
& D D_{o}=D D_{i}=D D_{s}=D D_{d}
\end{aligned}
$$

When no transform was performed, the admittances of the amplifier $Y_{i}, Y_{o}, Y_{s}, \Delta$ are written as equation (8). The property (7) is true.

$$
\begin{aligned}
Y_{i} & =G_{i}+s C_{i} \\
Y_{o} & =G_{o}+s C_{o} \\
Y_{s} & =(C i+C o+C r+C f) s \\
& +(G i+G o+G r+G f)
\end{aligned}
$$

$$
\begin{aligned}
\Delta & =(C i C o-C r C f) s^{2} \\
& +(G i C o+C i G o-G r C f-C r G f) s \\
& +(G i G o-G r G f)
\end{aligned}
$$

Let us assume that the property is true from the $(n-1)^{\text {th }}$ reduction. If the $n^{\text {th }}$ reduction is the series transform, we have the following relation:

$$
\begin{aligned}
Y_{i}^{(n)}= & \frac{\frac{\frac{N N_{i}}{D N_{i}}}{\frac{N D_{i}}{D D_{i}}} \frac{N e}{D e}+\frac{\frac{N N_{d}}{D N_{d}}}{\frac{N D_{d}}{D D_{d}}}}{\frac{\frac{N N_{s}}{D N_{s}}}{\frac{D D_{s}}{D D_{s}}}+\frac{N e}{D e}}=\frac{\frac{N N_{i}}{D N_{i}} \frac{N e}{D e}+\frac{N N_{d}}{D N_{d}}}{\frac{N N_{s}}{D N_{s}}+\frac{N e}{D e} \frac{N D_{s}}{D D_{s}}} \\
= & \frac{\frac{N N_{i} N e D N_{d}+N N_{d} D N_{i} D e}{D N_{i} D e D N_{d}}}{\frac{N N_{s} D e D D_{s}+N e N D_{s} D N_{s}}{D N_{s} D e D D_{s}}}
\end{aligned}
$$

By performing the same calculus for the variables $Y_{o}, Y_{s}, \Delta$, we show that (7) is true for the series transform. The reproduction of this calculus for the 5 others transformations shows that (7) is true for all the transformations. Note that this demonstration for the set of variables $Y_{i}, Y_{r}, Y_{f}, Y_{o}$ is identical and the property (7) remains true, i.e. $N D_{i}=N D_{r}=$ $N D_{f}=N D_{o}$ and $D D_{i}=D D_{r}=D D_{f}=D D_{o}$.

The advantage of this representation may be easily understood on the following simple example, where we simply consider the particular case of the addition of two admittances.

$$
\begin{aligned}
Y_{1}+Y_{2} & =\frac{\frac{N N_{1}}{D N_{1}}}{\frac{N D_{1}}{D D_{1}}}+\frac{\frac{N N_{2}}{D N_{2}}}{\frac{N D_{2}}{D D_{2}}}=\frac{\frac{N N_{1}}{D N_{1}}+\frac{N N_{2}}{D N_{2}}}{\frac{N D_{1}}{D D_{1}}} \\
& =\frac{\frac{N N_{1} D N_{2}+N N_{2} D N_{1}}{D N_{1} D N_{2}}}{\frac{N D_{1}}{D D_{1}}}
\end{aligned}
$$

By taking the "numerator" of this expression, one obtains the following equation :

$$
\text { Char Poly }=N N_{1} D N_{2}+N N_{2} D N_{1}
$$

Let us start again the characteristic polynomial calculus, by using the standard representation of admittances, that is under the form of a rational function. Since the denominators are differents, i.e. $D N_{1} N D_{1} \neq D N_{2} N D_{2}$, the sum of these two admittances may be written as (13).

$$
\begin{aligned}
Y_{1}+Y_{2} & =\frac{N N_{1} D D_{1}}{D N_{1} N D_{1}}+\frac{N N_{2} D D_{2}}{D N_{2} N D_{2}} \\
& =\frac{N N_{1} D D_{1} D N_{2} N D_{2}+N N_{2} D D_{2} D N_{1} N D_{1}}{D N_{1} N D_{1} D N_{2} N D_{2}}
\end{aligned}
$$

By taking the numerator of this expression, the characteristic polynomial can be put under the following form:

Char Poly $=N N_{1} D D_{1} D N_{2} N D_{2}+N N_{2} D D_{2} D N_{1} N D_{1}$ 
The comparison between expressions (12) and (14) shows that writing the admittances under the classical form (13) hides the common factors in the numerators of admittances. Consequently, at each steps of calculus, roots are added to the numerator. These additional roots substantially increase the degree of the characteristic polynomial.

\section{Conclusion}

We have proposed in this paper a general method to analyse quartz oscillators. The use of formal calculus allows to fully automatize this analysis. The software that we have developed parses the input netlist of the oscillator, identify and perform the impedance transformations to reduce the initial structure to a canonical form.

Applying the general oscillation condition gives the symbolic form of the characteristic polynomial which can eventually be used to analyse the oscillator behavior. The direct use of the transformation equations lead to polynomials of very high degree. We have shown that it is possible to solve this problem through a change of variable and an adequate representation of the amplifier admittances.

\section{Acknowledgments}

This work has been supported by CNES and DGA under contracts: \#832/CNES/88/5374/00, \# 962/CNES/91/1476/00, \# 962/CNES/94/1230/00.

\section{References}

[1] R. Brendel, F. Djian, E. Robert, High precision nonlinear computer modelling techniques for quartz crystal oscillators, Proc. 45th AFCS (1991).

[2] R. Brendel, G. Marianneau, T. Blin, M. Brunet, Computer aided design of quartz crystal oscillators, IEEE Trans. on UFFC, vol. 42, n. 4, pp. 700-708 (1995).

[3] R. Brendel, N. Ratier, L. Couteleau, G. Marianneau, P. Guillemot, Transient simulation in quartz crystal oscillators, Proc. of 11th EFTF (1997).

[4] M.E. Frerking, Crystal oscillator design and temperature compensation, Van Nostrand Reinhold Co (1978).

[5] L.W. Nagel, SPICE 2 : A computer program to simulate semiconductor circuits, Memorandum ERL-M520, Univ. of California, Berkeley (1975).

[6] N. Ratier, R. Brendel, T. Blin, G. Marianneau, P. Guillemot, Non linear simulation of quartz crystal oscillators, Proc. of 9th EFTF, pp. 88-92, (1995).
[7] N. Ratier, R. Brendel, P. Guillemot, Quartz oscillators: deriving oscillation condition by symbolic calculus, Proc. of 10th EFTF, pp. 442-446, (1996).

[8] P. Tuinenga, SPICE - A guide to circuit simulation and analysis using PSpice, 2nd edition, Prentice Hall, 1992. 